\title{
Utilizing Sidelobe ASK Based Joint Radar-Communication System Under Fading
}

\author{
Muharrem Arik and Ozgur B. Akan \\ Next-generation and Wireless Communications Laboratory (NWCL) \\ Department of Electrical and Electronics Engineering, Koc University, Istanbul, Turkey \\ Email: \{marik,akan\}@ku.edu.tr
}

\begin{abstract}
A joint radar-communication (JRC) system can provide cost-effective and spectrum-efficient platform solution with simultaneous operation, while accomplishing important tasks, sensing via radar processing and allocation of communication links. Existing modulation techniques where information embedding is achieved using sidelobe Amplitude-Shift Keying (ASK) for the JRC system are not investigated so far under fading channels and an optimum threshold estimation algorithm is yet to be developed. Specifying an optimum threshold level under fading can become a comprehensive problem, especially for mobile communication systems. In this paper, a novel non-data aided (NDA) threshold estimation technique and a receiver design are introduced. Furthermore, a new sidelobe ASK modulation technique is proposed for utilizing JRC system for mobile platforms under fading. Proposed modulation technique implements dual Sidelobe Level (SLL) ASK with waveform diversity by exploiting multiple orthogonal waveforms. One pair is modulated with dual SLL in amplitude rotational manner and initiates NDA threshold estimation process at the receiver. This method utilizes $K$ bits of information using only $K+1$ orthogonal waveform. The performance of the proposed technique is investigated in terms of the bit error rate (BER) and data rate. Simulations reveal that the operation of proposed method coupled with NDA threshold estimation process can reach more data rate, since it exhibits almost the same BER performance as existing methods under fading channel without requiring more orthogonal waveform.
\end{abstract}

\section{INTRODUCTION}

Wireless communication and radar always seek for different motivations. One focuses on achieving maximum capacity under noisy channel, the other searches for better target resolution and parameter estimation when dealing with clutter and noise. Furthermore, extensive efforts on spectrum management and efficiency against radio frequency spectrum congestion enable development of smart systems as cognitive radio [1] and radar [2], as well as radar and communication co-existence [3]-[5]. In recent years, huge attention is attracted with integration of communication and radar within the same system. A JRC system can provide cost-effective platform solution with simultaneous operation, while achieving important tasks, as environment sensing via radar processing and allocation of communication links. Furthermore, a JRC system can be a promising approach for cognitive radar networks [2], [6][8] to enable faster switching between radar waveforms, since it does not require any other communication system which may results some delay. Hence, any radar state can be shared between radar nodes from CPI to CPI.

Burying information into radar waveform via emitting one waveform during each radar pulse from a group of predetermined waveforms has been studied in [9]. According to which waveform is transmitted and received, the information is deciphered at the receiver. However, in each coherent processing interval (CPI) of radar, shifting the waveform from pulse to pulse basis may disturb the primary radar operation. Recently, a joint radar communication approach using time modulated arrays (TMA), named as dual function radar-communication (DFRC), was proposed in [10]. Later, waveform diversity (WD) was integrated and exploits the communication performance with ASK, coherent and non-coherent PSK methods [11]-[16]. All these solutions require a radar in multiple-input multiple-output (MIMO) mode, i.e. simultaneously transmitting orthogonal waveforms. Furthermore, they require off-line computations to reach corresponding transmit beamforming weights for establishing a communication channel at specific direction.

Recent researches in [17] and [18] enables real-time computation mechanism for updating the transmit beamforming vector to follow a mobile platform with low complexity procedures. Hence, in order to build mobile JRC systems, sidelobe ASK based JRC system has to be investigated under fading channels.

In this paper, a novel non data aided (NDA) threshold estimation technique is proposed. Pre-selected dual waveform feature eliminates the redundant training signal from the threshold estimation problem and exploits the proposed method for faster convergence to an optimum threshold. Furthermore, a receiver design is introduced and investigated for existing sidelobe ASK modulation schemes under fading channel. More to that, a new sidelobe ASK modulation technique is proposed for utilizing JRC system for mobile platforms. Proposed modulation technique implements dual Sidelobe Level (SLL) ASK by exploiting multiple orthogonal waveforms. One pair is modulated with dual SLL with amplitude rotational manner and this pair initiates NDA threshold estimation process at the receiver. This method utilizes $K$ bits of information using only $K+1$ orthogonal waveform.

The remainder is organized as follows. The signal model and transmit waveform design methods are described in Sec. II and Sec. III, then the proposed information embedding scheme is presented in Sec. IV. The threshold estimation procedures are detailed in Sec. V. Sec. VI provides the simulation results and conclusions are discussed in Sec. VII. 


\section{SignAl MOdEL}

In a DFRC system, the main mission of the transmit array is radar operation. However, it has a secondary purpose as burying information into radar waveforms toward the direction of communication without any effect on the radar operation [13]. The JRC system is equipped with $M$ transmit antennas aligned as a uniform linear array (ULA). The $M \times 1$ baseband transmit signal vector during the $n$th radar pulse is,

$$
s(t ; n)=\sqrt{\frac{P_{t}}{J+1}} \sum_{j=0}^{J} \mathbf{w}_{j}^{*}(n) \varphi_{j}(t),
$$

where $\mathbf{w}_{j}$ be the $M \times 1$ transmit beamforming weight vectors, where $n$ is the pulse index, $t$ is the fast time index, $(.)^{*}$ stands for the complex conjugate, $\varphi_{j}(t)$ be a set of $J+1$ orthogonal waveforms, each occupying the same bandwidth $B w$. The bandwidth for joint radar-communication functions is denoted as $B w$ and the total transmit power budget is $P_{t}$. In other words, the spectral contents of all waveforms overlap in the frequency domain. It is assumed that each waveform is normalized to have unit power, i.e. $\int_{T_{P W}}\left|\varphi_{j}(t)\right|^{2} d t=1, j=0,1, \ldots, J$, where $T_{P W}$ is the radar pulse width. It is further assumed that the orthogonality condition, $\int_{T_{P W}} \varphi_{j}(t) \varphi_{j^{\prime}}^{*}(t) d t=0, j=$ $0,1, \ldots, J$ is satisfied for $j \neq j^{\prime}$. Note that, in practice, perfectly orthogonal coded waveforms cannot be achieved. Therefore, the problem of waveform design with very low cross-correlations and very high auto-correlations should be used for this purpose and however, this is out of the scope of this section.

At the receiver side, a single omni-directional antenna element connected to a communication receiver located in direction $\theta_{c}$, which is known to the transmitter side. The baseband signal at the output of the communication receiver can be expressed as,

$$
y(t ; n)=\beta_{c h}(n) \mathbf{a}^{T}\left(\theta_{c}\right) s(t ; n)+\eta(t ; n)
$$

where $\mathbf{a}\left(\theta_{c}\right)$ is the $M \times 1$ steering vector of the transmit array toward the spatial angle $\theta_{c}, \beta_{c h}(n)$ is the channel coefficient which reflects the propagation gain between the transmit array and the communication receiver during the $n$th pulse, and $\eta(t ; n)$ is the additive white Gaussian noise with zero mean and variance $\sigma_{N}^{2}$. Using signalling strategies to construct the transmit signal vector $s(t ; n)$, communication symbols can be buried into the radar signal.

\section{Transmit BEAmForming DESIGN FOR SiDELOBE ASK}

First, transmit beamforming vectors for sidelobe level modulation are smartly selected to satisfy to focus the transmit power within the radar main beam, while keeping the sidelobe level below a certain value.

Off-line methods in the literature uses genetic algorithms [10] and second-order cone programming [13]. Transmit weight vectors can be designed off-line solving the following optimization problem from [19],

$$
\begin{gathered}
\min _{u_{k}} \max _{\theta}|| G_{d}(\theta)|-| \mathbf{u}_{k}^{H} \mathbf{a}(\theta)||, \theta \in \Theta, \\
\text { s.t. }\left\|\mathbf{u}_{k}^{H} \mathbf{a}(\theta)\right\| \leq \varepsilon, \theta \in \bar{\Theta}, \\
\mathbf{u}_{k}^{H} \mathbf{a}\left(\theta_{c}\right)=G_{k},
\end{gathered}
$$

where $\mathbf{u}_{k}$ be the $M \times 1$ transmit beamforming weight vectors for amplitude modulation, and $G_{k}$ is selected from a set of SLL symbols $\tilde{G}=\left\{G_{1}, G_{2}, \ldots, G_{K}\right\}$ is the desired transmit beam-pattern and $\Theta$ is the spatial sector where main radar operation takes place and $\bar{\Theta}$ sidelobe sector, $(.)^{H}$ stand for the Hermitian operations, $\varepsilon$ is a positive number that specify maximum SLL for primary radar operation, and $G_{k}$ corresponds to the $k$ th SLL towards the communication direction,

$$
\mathbf{a}(\theta)=\left[1, e^{-j 2 \pi d_{1} \sin (\theta)}, \ldots, e^{-j 2 \pi d_{M-1} \sin (\theta)}\right]^{T}
$$

is the $M \times 1$ transmit array steering vector toward the spatial angle $\theta,(.)^{T}$ stand for the transpose operations, $d_{m}$ is the displacement measured in wavelength between the $m$ th and $m+1$ th antennas where $m=0, \ldots, M-1$, respectively. This optimization problems are convex and can be efficiently solved using second-order cone programming as stated in [13].

Recent research in [18] proposes a closed form solution to an optimization procedure based on eigenvalue, point and derivative constraints. This method yields a low-complexity and robust solution for designing transmit beamforming vector in the JRC system. Hence, transmit weight vectors can be updated on-line calculating the following equation from [18],

$$
\begin{aligned}
\mathbf{u}_{k}\left(\theta_{c}\right)= & \mathbf{u}_{o}-\mathbf{U} \mathbf{U}^{H} \mathbf{A}_{\theta_{c}}\left(\mathbf{I}_{2}-\mathbf{Z}_{\theta_{c}} \mathbf{A}_{\theta_{c}}^{H}\left(\mathbf{U} \mathbf{U}^{H}\right)^{H} \mathbf{A}_{\theta_{c}}\right) \\
& \left(\mathbf{A}_{\theta_{c}}^{H} \mathbf{A}_{\theta_{c}}\right)^{-1} \mathbf{F}_{\theta_{c}}-\mathbf{A}_{\theta_{c}} \mathbf{Z}_{\theta_{c}} \mathbf{F}_{\theta_{c}}
\end{aligned}
$$

where $\mathbf{u}_{o}$ is the orginal given beamforming vector and,

$$
\begin{aligned}
\mathbf{F}_{\theta_{c}} & =\left[\mathbf{u}_{o}^{H} \mathbf{a}\left(\theta_{c}\right)-G_{k}, \mathbf{u}_{o}^{H} \tilde{\mathbf{a}}\left(\theta_{c}\right)\right]^{T} \\
\mathbf{Z}_{\theta_{c}} & =\left(\mathbf{A}_{\theta_{c}}^{H} \mathbf{U} \mathbf{U}^{H} \mathbf{A}_{\theta_{c}}-\mathbf{A}_{\theta_{c}}^{H} \mathbf{A}_{\theta_{c}}\right)^{-1}
\end{aligned}
$$

where $\mathbf{U}$ is the matrix whose columns are the eigenvectors of $\mathbf{Q}$ and $\mathbf{Q}=\int_{\theta_{c} \in \bar{\Theta}} \mathbf{a}\left(\theta_{c}\right) \mathbf{a}^{H}\left(\theta_{c}\right) d \theta_{c}, \mathbf{A}_{\theta_{c}}=\left[\mathbf{a}\left(\theta_{c}\right), \tilde{\mathbf{a}}\left(\theta_{c}\right)\right]$ and $\tilde{\mathbf{a}}\left(\theta_{c}\right)$ is the derivative of $\mathbf{a}\left(\theta_{c}\right)$.

Hence, if the receiver is mobile relative to the transmitter through the $\Theta$ axis, $\mathbf{u}_{k}\left(\theta_{c}+\theta_{\Delta}\right)$ can be updated with only changing $\mathbf{A}_{\theta_{c}+\theta_{\Delta}}$ and $\mathbf{F}_{\theta_{c}+\theta_{\Delta}}$ matrices as,

$$
\begin{gathered}
\mathbf{A}_{\theta_{c}+\theta_{\Delta}}=\mathbf{A}_{\theta_{c}} \odot \mathbf{A}_{\theta_{\Delta}} \\
\mathbf{F}_{\theta_{c}+\theta_{\Delta}}=\left[\mathbf{u}_{o}^{H}\left[\mathbf{a}\left(\theta_{c}\right) \odot \mathbf{a}\left(\theta_{\Delta}\right)\right]-G_{k}, \mathbf{u}_{o}^{H}\left[\tilde{\mathbf{a}}\left(\theta_{c}\right) \odot \mathbf{a}\left(\theta_{\Delta}\right)\right]\right]^{T}
\end{gathered}
$$

where $\odot$ is the Hadamard element-wise product. 


\section{Proposed Information Embedding Methods}

For each radar pulse, let $\chi_{b}=\left\{b_{1}(n), b_{2}(n), \ldots, b_{L}(n)\right\}$ sequence of binary bits buried into each radar pulse, then bits are smartly allocated for each orthogonal waveform using only two transmit beamforming vectors as $\mathbf{u}_{H}$ and $\mathbf{u}_{L}$.

The transmit beamforming vectors as $\mathbf{u}_{H}$ and $\mathbf{u}_{L}$ are assumed to have same transmit radiation pattern within an only exception is at the communication direction where SLL of $\mathbf{u}_{H}$ is higher than $\mathbf{u}_{L}$. Calculating the closed form equations in (79) while designing two weight vectors result $G_{k}=G_{H}$ for $\mathbf{u}_{H}$ and $G_{k}=G_{L}$ for $\mathbf{u}_{L}$.

\section{A. Dual Waveform Driven Multi Waveform ASK}

Let $J+1$ orthogonal waveforms is utilized for ASK modulation. For each radar pulse, let $\chi_{b}$ sequence of binary bits are grouped in $L$ and all is buried into each radar pulse where $J=L$. Fig. 1 shows the detailed dual waveform driven waveform allocation table. First two orthogonal waveform is selected to delivering first bit. Dual waveform pair, $\varphi_{0}(t)$ and $\varphi_{1}(t)$, is non-coherently modulated with $G_{H}$ and $G_{L}$ with rotational manner. This pair initiates NDA threshold estimation which will be detailed in Section $\mathrm{V}$ process at the receiver. The other waveforms are modulated with $G_{H}$ when $b_{l}(n)=1$ and $G_{L}$ when $b_{l}(n)=0$, for $l=2, \ldots, L$. Then, the baseband transmit signals in (1) can be rewritten as,

$$
\begin{aligned}
s(t ; n)= & P_{\Delta}\left\{\left[b_{1} \mathbf{u}_{L}^{*}+\left(1-b_{1}\right) \mathbf{u}_{H}^{*}\right] \varphi_{0}(t)\right. \\
& \left.+\sum_{l=1}^{L}\left\{\left[b_{l} \mathbf{u}_{H}^{*}+\left(1-b_{l}\right) \mathbf{u}_{L}^{*}\right] \varphi_{l}(t)\right\}\right\},
\end{aligned}
$$

where $P_{\Delta}=\sqrt{\frac{P_{t}}{L+1}}$ and the baseband representation of the received signal vector at the receiver is given by,

$$
\begin{aligned}
y(t ; n)= & P_{\Delta} \beta_{c h}(n)\left\{\left[b_{1} G_{L}+\left(1-b_{1}\right) G_{H}\right] \varphi_{0}(t)\right. \\
& +\sum_{l=1}^{L}\left\{\left[b_{l} G_{H}+\left(1-b_{l}\right) G_{L}\right] \varphi_{l}(t)\right\}+\eta(t ; n)
\end{aligned}
$$

where $G_{H}(n)=\mathbf{u}_{H}^{H} \mathbf{a}\left(\theta_{c}\right)$ and $G_{L}(n)=\mathbf{u}_{L}^{H} \mathbf{a}\left(\theta_{c}\right)$ are complex transmit gains towards $\theta_{c}$. After matched filtering with the coefficients of $\varphi_{0}, \varphi_{1}$ and $\varphi_{l}$, the received signal in (13) becomes,

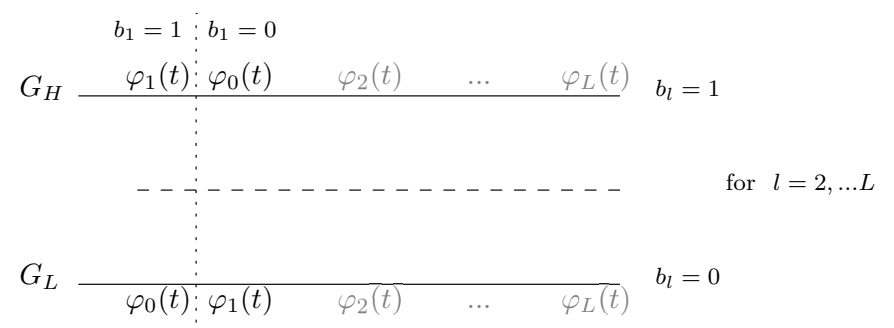

Fig. 1. Dual Waveform Driven Waveform Allocation Table

$$
\begin{aligned}
& y_{0}(n)=P_{\Delta} \beta_{c h}(n)\left[b_{1} G_{L}+\left(1-b_{1}\right) G_{H}\right]+\sigma_{0}(n) \\
& y_{l}(n)=P_{\Delta} \beta_{c h}(n)\left[b_{l} G_{H}+\left(1-b_{l}\right) G_{L}\right]+\sigma_{l}(n)
\end{aligned}
$$

where $l=1, \ldots, L$.

The signal strength at the output of the matched filters, original symbols can be retrieved by doing the following multistage ratio test,

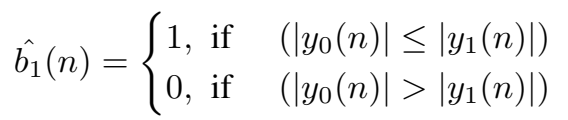

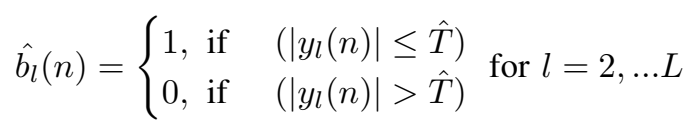

where $\hat{T}$ is the threshold level which is estimated with the method explained in Section V. Then, the receiver outputs the information bits using the formulas $(15,16)$ to retrieve the binary sequence $\chi_{b}$.

\section{Non-Data Aided (NDA) Threshold Estimation METHODS}

In this section, NDA threshold estimation methods which do not require an extra training signal to estimate the channel parameters are detailed. First, a time average NDA threshold estimation algorithm is introduced for the method in [14] for fading channels. Then, dual waveform driven (DWD) NDA algorithm which is designed for the method in Sec.IV is specified. Note that, data aided (DA) channel equalization techniques, i.e. channel sounding or sensing, can be applied, however they would disrupt or disturb the main radar operation or decrease the data rate. Also, if the number of user is increased in a radar network, it is harder to maintain the DA process for each node.

We assume that the channel is almost constant at least over the number of radar pulses used for observation, $N_{\text {obs }}$. Optimal threshold level can be defined as an intercept point of two rician distribution, $R\left(y_{l} \mid \Delta_{H}, \sigma_{l}^{2}\right)$ and $R\left(y_{l} \mid \Delta_{L}, \sigma_{l}^{2}\right)$, where $\Delta_{H}=\sqrt{\frac{P_{t}}{L+1}} \beta_{c h}(n) G_{H}$ and $\Delta_{L}=\sqrt{\frac{P_{t}}{L+1}} \beta_{c h}(n) G_{L}$ [13]. $y_{l}$ is a random variable with Rician distribution, since $\beta_{c h}(n)$ is almost constant for $N_{o}$ pulses and $\sigma_{l}(n)$ is zeromean Gaussian noise in (14).

\section{A. Time-Average NDA Threshold Estimation}

It was shown that thresholds for the symbol decision can be estimate without using redundant signals for PAM signals in [20]. In this research, the assumption is that, if the received SNR is much larger than the multiplication of symbol period and receiver bandwidth, in a observation period seeing the channel factor into deep fades was little probable. Therefore, the threshold is calculated with the time-average estimate.

In a DFRC system, due to waveform diversity feature, all waveforms have to be transmitted at the same time. Therefore, all the waveforms are affected by the same channel factor. Hence, this method stands as a promising approach for the 
high PRF systems that run over slow fading channel environments.

The time-average NDA threshold estimation algorithm is in two stage. At the first stage initial threshold is estimated using the all waveforms matched filter outputs with the method in [20] as,

$$
\bar{T}_{w}=\frac{1}{N_{o}(L+1)} \sum_{n=w-N_{o}+1}^{w} \sum_{l=0}^{L}\left|y_{l}(n)\right|,
$$

where $w$ is the any radar pulse index as $n=0,1, . ., w, . ., N$. Then, the estimated threshold, i.e. $\bar{T}$, is refined recursively based on decision-directed estimation method as,

$$
\begin{aligned}
\hat{T}_{w}= & \frac{1}{2 N_{\text {ones }}} \sum_{n=w-N_{o}+1}^{w} \sum_{l=0}^{L} \mid y_{l}\left(n \mid \hat{b}_{l}=1 \text { using } \bar{T}_{w}\right) \mid+ \\
& \frac{1}{2 N_{\text {zeros }}} \sum_{n=w-N_{o}+1}^{w} \sum_{l=0}^{L} \mid y_{l}\left(n \mid \hat{b}_{l}=0 \text { using } \bar{T}_{w}\right) \mid,
\end{aligned}
$$

where $N_{\text {ones }}$ and $N_{\text {zeros }}$ is the number of bits detected as 1 or 0 using $\bar{T}_{w}$, respectively. For each radar pulse, $\hat{T}$ is recursively updated using decision directed method. Then, $\hat{T}_{w+1}$ can be found by replacing $w$ as $w+1$ and $\bar{T}_{w}$ as $\hat{T}_{w}$ from the equation 18.

Furthermore, we apply this algorithm to the method in [14] and evaluate its performance in Section VI-B.

\section{B. Dual Waveform Driven (DWD) Threshold Estimation}

DWD Sidelobe ASK method comes with an important feature that each radar pulse contains at least one of the both SLL. Hence, this feature is exploited by the proposed NDA method for faster convergence to optimum threshold which results better BER.

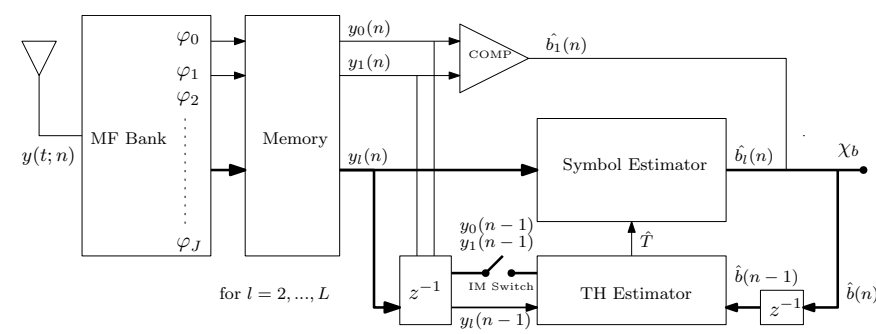

Fig. 2. Dual Waveform Driven Non-Data Aided Threshold Estimator.

A block diagram of the receiver model using DWD method is shown in Fig.2. Model consists of the matched filter bank (MF Bank), memory and comparator blocks, NDA threshold estimator (NDA TH Estimator), symbol estimator and appropriate delay blocks. At the first stage of the algorithm, matched filter outputs of dual waveforms, $\varphi_{0}$ and $\varphi_{1}$, is used to determine initial mode (IM) threshold level with using the IM switch on in Fig.2. Then, first stage symbols are estimated using the initial threshold. Initial threshold is a time average estimate of the matched filter output of $\varphi_{0}$ and $\varphi_{1}$ using (17) for $l=0,1$. At the second stage, using this initial threshold, $b_{l}(\hat{w})$ can be found using the (16), then threshold $\bar{T}$ is refined as decision-directed manner in (18).

According to the communication environment, $N_{o b s}$ should be selected smartly. For slow fading conditions, $N_{\text {obs }}$ can be selected as big enough to reach optimum threshold, hence smaller number of radar waveform can be used for modulation. On the other hand, fast fading channel conditions, in order to not to converge erroneous threshold, $N_{o b s}$ should be as small as possible. Then, if the number of radar waveform is big enough, algorithm converges to optimal threshold level again.

\section{Performance Evaluation}

In this section, simulation results are presented evaluating the communication performance of the proposed method for Sidelobe ASK based DFRC system in terms of bit error rate (BER). Then, we investigate the proposed threshold estimation method performance in terms of BER for various window sizes.

A uniform linear transmit array with 10 antennas spaced with one-half wavelength is used at the simulations. During each radar pulse, information bits are transmitted towards the communication direction located in the sidelobe region. For all methods, only two transmit vectors, $\mathbf{u}_{H}$ and $\mathbf{u}_{L}$, are used for modulation to provide $-20 \mathrm{~dB}$ and $-40 \mathrm{~dB}$ communication SLL. The transmit beampatterns for these vectors are given in Fig.3 using the formulation in [18].

In order to reach results, a Monte Carlo approach has been used over 100 tests. For each test, a sequence of $10^{4}$ radar pulses were transmitted to the channel. The communication channel is modelled as rician fading channel. We assumed that there is at least one line of sight (LOS) path exist. The ratio between the power of the direct path and the others, i.e. rician factor, was selected as 5 and channel coherence time is selected as $1.4 \mathrm{msec}$, unless otherwise stated.

Throughput is calculated as 80bits per packet with uncoded $64 b i t s$ data, 8bits checksum and 8bit header. We assume that receiver side operates in a simple ARQ mechanism and sends an ACK if any error is encountered.

\section{A. Proposed DWD ASK Modulation Performance}

We first investigate the performance of proposed method under additive white gaussian noise (AWGN) and rician fading

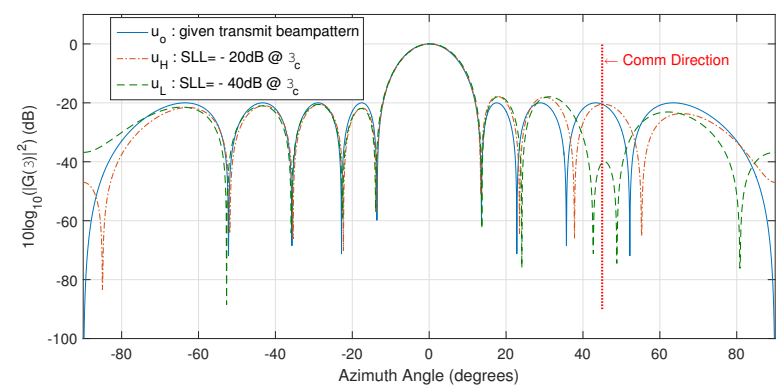

Fig. 3. Transmit beampatterns with Sidelobe ASK modulation using the method in [18] 


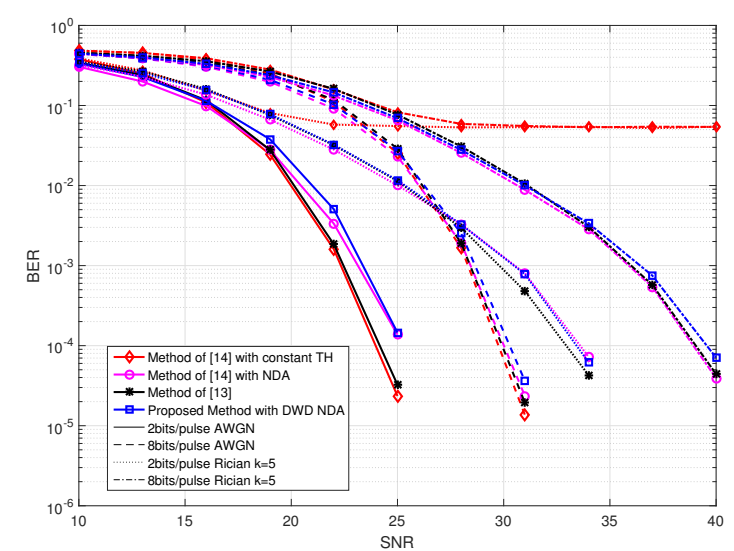

(a)

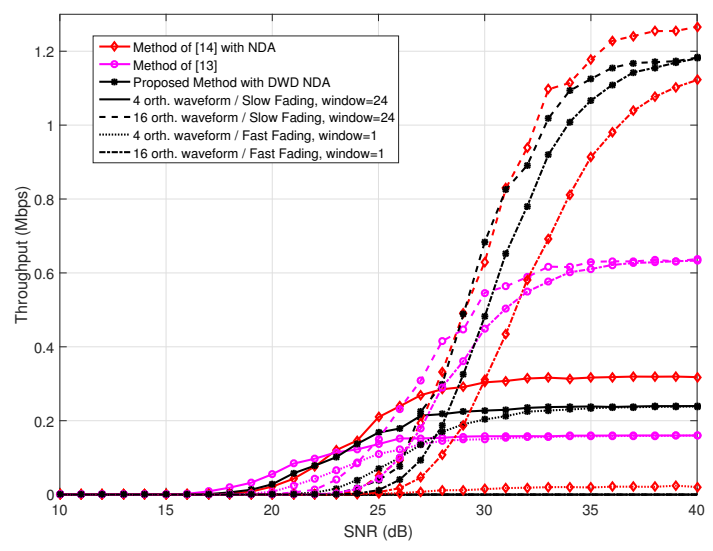

(b)

Fig. 4. Performance Comparison (a) BER vs SNR (fixed data rate) (b) Throughput vs SNR (fixed no. of orth. waveform)

channel in terms of BER for various SNR levels comparing with the existing schemes in [13] and [14]. Simulations are run for $L=2$ and $L=8$ bits delivered per pulse. For these cases, the number of orthogonal waveform $J$ is selected as, 3 and 9 for the proposed method, 2 and 8 for the methods in [13] and 4 and 16 for in [14], respectively. Hence, the window size is selected as $N_{o b s}=24$ pulses.

As expected, under AWGN channel and 2bits/pulse case, the proposed methods using NDA threshold mechanism show $1 d B$ behind BER performance. Hence, the window size were not enough to converge optimum threshold level. For the 8bits/pulse case, almost 4 times higher samples are used for the threshold estimation process. Then, the proposed methods using NDA threshold mechanism perform almost same BER performance as the others.

As in Fig.4(a), all the methods show almost the same performance under rician fading, however the method of [13] has the slightly the best BER performance. Hence, it does not require any threshold mechanism. However, it needs twice the number of orthogonal waveform to reach the required data rate. On the other hand, the proposed method with DWD NDA algorithm has better BER performance than the method

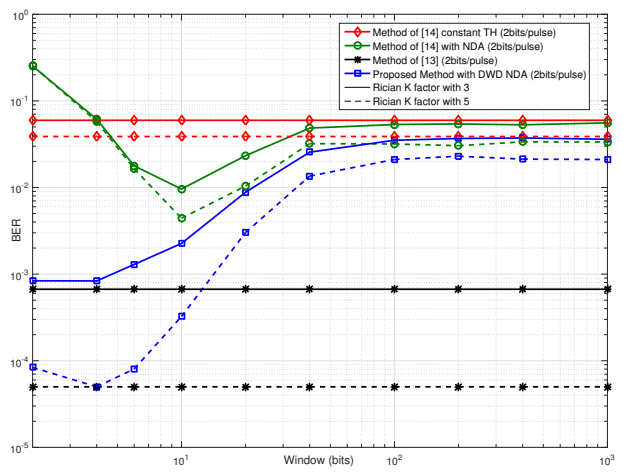

(a)

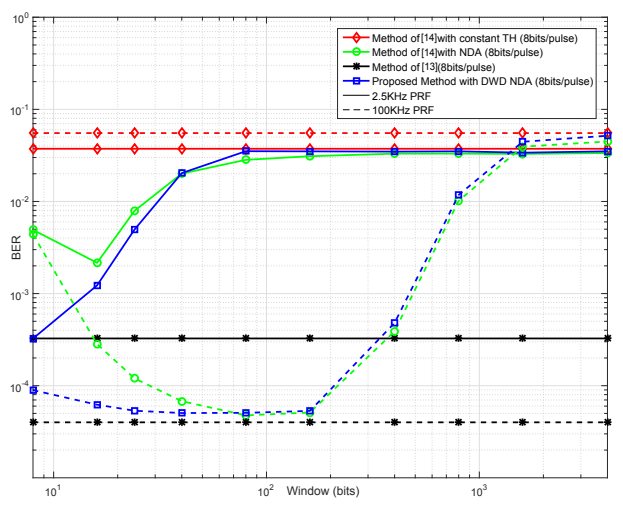

(b)

Fig. 5. Dual Waveform Driven NDA Threshold Estimation Algorithm Performance for (a) 2 bits/pulse and (b) 8 bits/pulse vs Window Size $\left(N_{o b s} \times L\right)$ when $\mathrm{PRF}=10 \mathrm{KHz}$ and Rician $\mathrm{K}=5$

of [14], although proposed method requires one additional orthogonal waveform.

In the second part of the simulations, we evaluated the system throughput under changing fading conditions, while the number of orthogonal waveform keeps the same as in Fig.4(b). Keeping the waveform fixed is clearly the more realistic case, because the DFRC system is developed with a claim that the system would not cause any effect on radar mission.

As seen in Fig.4(b), the method of [13] has the lowest capacity and [14] with proposed NDA solution has the highest. Simulations show that, the method of [13] reaches higher throughput under only low SNR case and it is not dependent to slow/fast fading case. On the other hand, proposed method with DWD NDA algorithm works best under fast fading and slightly higher SNR. The results are summarized in Table I. In this part of the simulations, the fast fading condition means the coherence time of the channel is smaller then the pulse repetition interval (PRI) and for the slow fading case, it is much greater than the PRI.

\section{B. Threshold Estimation Method Performance}

The performance of proposed threshold estimation method under rician channel in terms of BER for various window sizes $N_{o b s} \times L$ (in bits) is compared with existing modulation schemes. We are trying to show the convergence performance 
TABLE I

APplicABle Operating Conditions For Sidelobe ASK BASED JRC

\begin{tabular}{c|cc|ccc} 
Parameters & \multicolumn{2}{|c|}{ Channel } & \multicolumn{3}{c}{ Applicable Methods } \\
\hline \# $\boldsymbol{o f} \boldsymbol{W} \boldsymbol{v} \boldsymbol{m} \boldsymbol{m}$. & $\boldsymbol{S N R}$ & $\boldsymbol{F a d i n g}$ & {$[\mathbf{1 3}]$} & {$[\mathbf{1 4}] / \boldsymbol{N D A}$} & $\boldsymbol{D W D} / \boldsymbol{N D A}$ \\
\hline Low & Low & Slow & $\checkmark$ & $\times$ & $\times$ \\
\hline Low & Low & Fast & $\checkmark$ & $\times$ & $\times$ \\
\hline Low & High & Slow & $\times$ & $\checkmark$ & $\times$ \\
\hline Low & High & Fast & $\times$ & $\times$ & $\checkmark$ \\
\hline High & Low & Slow & $\checkmark$ & $\times$ & $\times$ \\
\hline High & Low & Fast & $\checkmark$ & $\times$ & $\times$ \\
\hline High & High & Slow & $\times$ & $\checkmark$ & $\times$ \\
\hline High & High & Fast & $\times$ & $\times$ & $\checkmark$ \\
\hline \multicolumn{7}{r}{} & & & &
\end{tabular}

of the proposed NDA methods to the optimal threshold level. Simulations are run for 2 and 8 bits of information delivered per radar pulse and observation window, which is selected through 1 to 500 radar pulses.

As in Fig.5(a), the proposed method with DWD NDA threshold estimation algorithm shows the same BER performance with [13], although the channel has more strongest fading effect.

Fig.5(b) displays the effect of the PRF on the BER performance. The method in [13] shows a reference BER performance due to its threshold-less mechanism. Hence, it specifies an baseline for other methods. On the other hand, the method in [13] requires twice times higher number of orthogonal waveform for the same number of bits, hence it modulates a bit with a pair of orthogonal waveform. The proposed method with DWD NDA algorithm reaches almost the same BER performance for window size 1 pulse and then increasing window size results setting the threshold to a wrong level, hence the channel is no longer constant along the observation window. In other words, the higher PRF means more pulse to sent in channel coherence time. Besides, the performance of the method in [14] exhibits the worst performance, since time-average NDA algorithm can not observe any relatively constant channel for converging to an optimal threshold level.

\section{CONCLUSIONS AND FUture WORK}

A JRC system can provide cost-effective platform solution while achieving environment sensing via radar processing and allocation of communication links. In this paper, a new sidelobe ASK modulation technique coupled with a NDA threshold estimation method for DFRC systems is developed. Furthermore, a new receiver architecture is introduced for utilizing DFRC system for mobile platforms.

Simulations show that the communication performance of the proposed and existing sidelobe ASK based modulation schemes are almost same under fading channel in terms of BER, while keeping data rate the same. Moreover, the operation of proposed DWD method coupled with NDA threshold estimation process drives higher throughput for fast fading and slightly higher SNR values. Besides, the proposed NDA algorithm makes the existing method in [14] delivering higher throughput for the slow fading case. In order to reach better performance at lower SNR values, instead of single antenna at the receiver, using an antenna array with digital beamforming will improve the performance significantly. For further investigation, different diversity combining techniques to mitigate multipath effects will be analysed.

\section{REFERENCES}

[1] A. J. Goldsmith and L. J. Greenstein, "Principles of Cognitive Radio," Cambridge, U.K.: Cambridge Univ. Press, 2012.

[2] S. Haykin, X. Sue and P. Stoodeh, "Cognitive Radar: Step Toward Bridging the Gap Between Neuroscience and Engineering," in proc. of the IEEE, November 2012.

[3] C. Sturm, W. Wiesbeck, "Waveform Design and Signal Processing Aspects for Fusion of Wireless Communications and Radar Sensing," in Proc. of the IEEE, vol. 99, no. 7, pp. 1236-1259, July 2011.

[4] G. Lellouch, H. Nikookar, "On the Capability of a Radar Network to Support Communications," Communications and Vehicular Technology in the Benelux, 2007, 14th IEEE Symposium on, pp. 1-5, Nov. 2007.

[5] S.D. Blunt, P. Yatham, and J. Stiles, "Intrapulse radar-embedded communications," Aerospace and Electronic Systems, IEEE Transactions on, vol. 46, no. 3, pp. 11851200, July 2010.

[6] S. Haykin, "Cognitive radar networks," in Proc. IEEE International Workshop on Computational Advances in Multi-Sensor Adaptive Processing, Dec. 2005, pp. 13.

[7] S. Haykin, "Cognitive radar networks," in Proc. Fourth IEEE Workshop on Sensor Array and Multichannel Processing, July 2006, pp. 124.

[8] S. Haykin, T. J. Nohara, "Cognitive Radar Information Networks for Security along Canada/U.S Border," White Paper prepared for DRDC CSS, Oct. 2012, www.accipiterradar.com/media/pdf/CIRNWhitepaper.pdf

[9] S. D. Blunt, M. R. Cook, and J. Stiles, "Embedding information into radar emissions via waveform implementation," in Proc. Int. Waveform Diversity \& Design Conf., Niagara Falls, Canada, Aug. 2010, pp. 813.

[10] J. Euziere, R. Guinvarc'h, M. Lesturgie, B. Uguen, R. Gillard, "Dual Function Radar Communication Time-Modulated Array," IEEE Intl. Radar Conf., 2014.

[11] A. Hassanien, M. G. Amin, Y. D. Zhang, and F. Ahmad, "Signaling strategies for dual-function radar-communications: An overview," IEEE Aerospace and Electronic Systems Magazine, In Press, 2016.

[12] A. Hassanien, M. G. Amin, Y. D. Zhang, and F. Ahmad, "PhaseModulation Based Dual-Function Radar-Communications," IET Radar, Sonar \& Navigations, 2016.

[13] A. Hassanien, M. G. Amin, Y. D. Zhang, and F. Ahmad, "Dual-function radar-communications: Information embedding using sidelobe control and waveform diversity," IEEE Trans. Signal Processing, vol. 64, no. 8, pp. 21682181, Apr. 2016.

[14] A. Hassanien, M. G. Amin, Y. D. Zhang, and F. Ahmad, "A dual function radar-communications system using sidelobe control and waveform diversity," in Proc. IEEE Int. Radar Conf. (RadarCon 2015), Arlington, VA, USA, May 2015.

[15] A. Hassanien, M. G. Amin, Y. D. Zhang, F. Ahmad, and B. Himed, "Non-coherent PSK-based dual-function radar-communication systems," in Proc. IEEE Radar Conf., Philadelphia, PA, May 2016.

[16] E. BouDaher, A. Hassanien, E. Aboutanios, and M. G. Amin, "Towards a dual-function MIMO radar-communication system," in Proc. IEEE Radar Conf., Philadelphia, PA, May 2016.

[17] A. de Oliveira, R. Sampaio-Neto, J. M. Fortes, "Robust Radar-Embedded Sidelobe Level Modulation using Constrained Optimization Design," in Proc. IEEE Radar Conf., Philadelphia, PA, May 2016.

[18] A. de Oliveira, R. Sampaio-Neto, J. M. Fortes, "Low Complexity Robust Radar-Embedded Sidelobe Level Modulation using Linear Constrained Optimization Design," in Proc. of the IEEE Sensor Array and Multichannel Signal Processing Workshop (SAM), Rio de Janerio, 2016.

[19] A. Hassanien, M. G. Amin, and Y. D. Zhangy, "Computationally Efficient Beampattern Synthesis for Dual-Function Radar-Communications," in Proc. SPIE Defence + Security, Radar Sensor Technology Conf., Baltimore, MD, April 2016.

[20] A. Anttonen, A. Kotelba and A. Mammela, "Nondata-Aided Hypothesis Testing of PAM Signals with Energy Detection," IEEE Communications Letters, vol. 14, no. 10, pp. 957959, October 2010. 